\title{
EXPRESSÃO DE p53, p16 E COX-2 EM CARCINOMA ESCAMOSO DE ESÔFAGO E ASSOCIAÇÃO HISTOPATOLÓGICA
}

\author{
Izabella Paz Danezi FELIN ${ }^{1,2}$, Ivana GRIVICICH ${ }^{1,3,4}$, Carlos Roberto FELIN ${ }^{1,5,6}$, \\ Andrea REGNER ${ }^{1,3,4}$ e Adriana Brondani da ROCHA ${ }^{1,3,4}$
}

\begin{abstract}
RESUMO - Racional - O câncer de esôfago representa cerca de 2\% dos tumores malignos e a terceira causa mais comum de câncer do trato gastrointestinal. A associação do prognóstico do câncer de esôfago com alguns marcadores imunoistoquímicos, como as proteínas p53, p16 e a ciclooxigenase 2 (COX-2) tem sido relatada. A detecção de marcadores moleculares através de imunoistoquímica pode ser utilizada para avaliação prognóstica. Objetivos - Investigar a associação entre a expressão das proteínas p53, p16 e a COX-2 com o estádio do carcinoma escamoso de esôfago. Métodos - Foram analisadas 31 amostras de ressecção cirúrgica por esofagectomia diagnosticadas como carcinoma de células escamosas de esôfago e 31 amostras não-tumorais referentes a cada caso. Realizou-se a revisão histopatológica e o estádio pTNM. Amostras tumorais e não-tumorais adjacentes foram submetidas a análise imunoistoquímica para avaliar o conteúdo das proteínas p53, p16 e COX-2. Foi considerada positiva a expressão nuclear para p53 em quantidade igual ou superior a 10,00\% das células e presença da expressão citoplasmática de acordo com três escores $(1,2,3)$ de intensidade (leve, moderada, acentuada) de imunocoloração para COX-2. Resultados - Em área tumoral, as análises revelaram 48,38\% de positividade para p53, 16,12\% de positividade para p16, e 100,00\% de positividade escores $1+, 2+$ ou $3+$ para COX-2. No entanto, quando se avaliou possível relação da expressão destes marcadores com o estádio, apenas a COX-2, escore 3+ intensidade acentuada mostraram associação significativa. Conclusão - O presente estudo demonstrou que existe relação positiva entre a expressão de COX-2, escore 3+ e estádio mais avançado no carcinoma de esôfago.
\end{abstract}

DESCRITORES - Neoplasias esofágicas. Carcinoma de células escamosas. Proteína supressora de tumor p53. Inibidor p16 de quinase ciclinadependente. Ciclooxigenase 2 .

\section{INTRODUÇÃO}

O câncer de esôfago representa cerca de $2 \%$ dos tumores malignos e a terceira causa mais comum de câncer do trato gastrointestinal. Aproximadamente 85\% dos cânceres de esôfago são carcinomas de células escamosas $^{(6,10)}$. A carcinogênese esofágica tem associação com vários fatores, incluindo: ambientais, estilo de vida, distúrbios esofagianos e agentes predisponentes como doença celíaca e raça negra ${ }^{(6)}$.

O diagnóstico de câncer de esôfago envolve suspeita clínica, quando são relatados sinais e sintomas, tais como disfagia progressiva e emagrecimento, odinofagia e dor retroesternal. $\mathrm{Na}$ investigação diagnóstica é essencial efetuar endoscopia digestiva alta com biopsia, seguida de exame histopatológico ${ }^{(3,21,31)}$. No caso de positividade tumoral, a ressecção da peça cirúrgica, com posterior análise histológica, incluindo graduação e estádio tumoral, feito através do sistema TNM, tem importância prognóstica e terapêutica ${ }^{(6,27)}$.
A carcinogênese esofágica envolve descontrole da proliferação, diferenciação e apoptose celular, observandose assim, alterações genéticas relacionadas com o controle desses processos ${ }^{(18)}$. A carcinogênese esofágica envolve múltiplas etapas, onde agentes carcinogênicos atuam provocando mutações no DNA, que podem ou não ser corrigidas pelos genes de reparo do DNA. Quando o dano ao DNA é intenso e o reparo é insuficiente, o mecanismo de apoptose é ativado e a célula é eliminada. A ativação do reparo e da apoptose pode ocorrer através da ação da proteína p53 selvagem que interrompe o ciclo celular, verifica possíveis danos do DNA e, se necessário, induz o reparo ou ativa o mecanismo de apoptose. Entretanto, quando ocorre mutação no gene p53, a proteína p53 torna-se não-funcional, levando a uma instabilidade genética e iniciando a transformação maligna ${ }^{(6,13,28)}$. De modo geral, as mutações, na tumorigênese, podem ocasionar ativação dos oncogenes, inativação de genes supressores tumorais e alterações dos genes de reparo do DNA $^{(4,6)}$. Os produtos dos genes mutantes são chamados

Trabalho realizado no Laboratório de Marcadores de Estresse Celular, Centro de Pesquisas em Ciências Médicas, Universidade Luterana do Brasil, Canoas, RS. ${ }_{1}^{1}$ Programa de Pós-graduação em Genética e Toxicologia Aplicada, Universidade Luterana do Brasil, Canoas, RS.; ${ }^{2}$ Departamento de Patologia, Universidade Federal de Santa Maria, RS.; ${ }^{3}$ Programa de Pós-graduação em Diagnóstico Genético e Molecular, Universidade Luterana do Brasil; ${ }^{4}$ Laboratório de Marcadores de Estresse Celular, Centro de Pesquisas em Ciências Médicas, Universidade Luterana do Brasil; ${ }^{5}$ Hospital da Brigada Militar de Santa Maria, RS.; ${ }^{6} \mathrm{Hospital}$ Universitário da Universidade Federal de Santa Maria. Correspondência: Dra. Adriana Brondani da Rocha - Laboratório de Marcadores de Estresse Celular, Centro de Pesa
do Brasil - Av. Farroupilha, 8001 - Prédio 22, 5ª andar - 92245-900 - Canoas, RS. E-mail: brondani@terra.com.br 
oncoproteínas e podem ser identificadas através do exame imunoistoquímico ${ }^{(22,26)}$

Neste sentido, a associação do prognóstico do câncer de esôfago com alguns marcadores imunoistoquímicos, tais como as proteínas p53, p16 e a ciclooxigenase 2 (COX-2) têm sido relatada $^{(18,20)}$. As proteínas p16, p21 e p53 são inibidoras do complexo $\mathrm{CDK}_{4 / 6}$ e ciclina D1, que é o complexo responsável pela fosforilação da proteína repressora $\mathrm{pRB}$, levando à parada do ciclo celular em $\mathrm{G} 1^{(7)}$.

A COX-2 é uma enzima presente em processos inflamatórios, responsável por sintetizar prostanóides a partir do ácido aracdônico, importante na conversão do ácido aracdônico em prostaglandina. É expressa numa série de tumores, principalmente os associados à inflamação e está envolvida no processo de carcinogênese e inibição da apoptose. Sua presença pode ser fator prognóstico independente, cujo nível de expressão tem sido relacionado ao desenvolvimento de metástases à distância, recurrências, menor sobrevida e grau de invasão vascular ${ }^{(8)}$.

No presente estudo, avaliou-se se existe associação entre a expressão das proteínas p53, COX-2 e p16 com o estádio do carcinoma, escamoso de esôfago.

\section{MÉTODOS}

\section{Seleção de casos}

Foram escolhidos blocos de parafina $(n=31)$ contendo amostras de tecido obtidas de produtos de ressecção por esofagectomia anteriormente diagnosticados como carcinoma de células escamosas de esôfago humano. Os blocos foram provenientes do arquivo do Serviço de Patologia do Hospital Universitário de Santa Maria (HUSM), RS, referentes aos exames histopatológicos rotineiros realizados no período entre 1998 e 2003. Amostras de áreas não-tumorais de mucosa adjacente, referentes a cada caso, foram incluídas no estudo $(n=31)$, para fins de comparação da expressão imunoistoquímica entre áreas não-neoplásicas e neoplásicas.

Como critério de inclusão, foi considerada a existência e a conservação adequada de todos os blocos de parafina referentes a cada caso no período de tempo destinado ao estudo. Foram considerados critérios de exclusão a má conservação dos blocos de parafina, arquivo incompleto, dúvidas diagnósticas, outros diagnósticos, produtos de biopsias, casos fora do período estipulado, casos tratados com quimioterapia e ou radioterapia neoadjuvante e casos com artefatos de fixação e processamento.

O protocolo experimental desenvolvido neste estudo foi submetido e aprovado pelo Comitê de Ética em Pesquisa em Seres Humanos e Animais da Universidade Luterana do Brasil (ULBRA) sob protocolo CEP-ULBRA 2006-266H.

\section{Processamento histológico}

Foram obtidos de todos os blocos cortes histológicos com $4 \mu \mathrm{m}$ de espessura, montados em lâminas histológicas tratadas com silano (4\%) e coradas com hematoxilina-eosina (H-E) para reavaliação histopatológica.
A revisão histopatológica em todos os casos foi realizada por dois patologistas experientes, de forma independente, sem identificação dos casos. Eventuais resultados conflitantes foram discutidos pelos mesmos para definição consensual da análise.

Procedeu-se à graduação, estádio pTNM e caracterização de itens relativos ao preenchimento de um instrumento recomendado para câncer esofágico ${ }^{(17)}$, adotado pelo Serviço de Patologia do HUSM.

Dos itens envolvidos na padronização do laudo anatomopatológico em esôfago, foi selecionado o estádio (pTNM), muito relacionado ao prognóstico em câncer de esôfago, confrontando-o com os resultados imunoistoquímicos. Também foram confrontadas as expressões das proteínas p53, p16 e COX-2 em tecido tumoral e não-tumoral.

\section{Imunoistoquímica}

Através de imunoistoquímica ${ }^{(2,11)}$ foram pesquisados, em todos os casos $(n=31)$ de carcinoma de esôfago e mucosa adjacente, as proteínas p53, COX-2 e p16 ${ }^{\text {ink } 4 a}$.

As lâminas contendo amostras tumorais e não-tumorais de esôfago foram desparafinizadas em xilol e hidratadas em álcool etílico absoluto. A seguir, uma lâmina de cada bloco foi corada pela técnica de H-E e outras lâminas foram desparafinizadas e imunocoradas pela técnica imunoistoquímica para o marcador específico.

Para todos os marcadores foram seguidos os passos de desparafinização e recuperação antigênica de acordo com as orientações dos fabricantes, para p53 (Dakocytomation/EUA), para COX-2 (Chemical Co.) e para p16 (Neomarkers/EUA). Resumidamente, as lâminas foram tratadas com tampão de ácido cítrico (pH 6,00) para reativação do antígeno e lavadas em solução salina tamponada. Após, foram tratadas com peróxido de hidrogênio $\left(\mathrm{H}_{2} \mathrm{O}_{2}\right)$ a $3 \%$ por 5 minutos, para bloqueio da peroxidase endógena. A seguir foram incubadas "overnight" a $4-8^{\circ} \mathrm{C}$ com os respectivos anticorpos primários: p53 (anticorpo monoclonal de camundongo, clone DO-7; DakoCytomation/EUA), na diluição 1:1800; COX-2 (anticorpo monoclonal de camundongo, clone CX229; Cayman Chemical Co./EUA), na diluição 1:100; p16 (anticorpo monoclonal de camundongo, clone AP4 (16PO4); Neomarkers/EUA).

Posteriormente todas as lâminas foram lavadas com PBS com Tween, aspirado o excesso e instilado o Envision Mouse (REF:4001-Dako). No sistema Envision, o anticorpo secundário (anti-camundongo) e sistema de detecção foram incubados em um só passo da reação imunoistoquímica por 45 minutos, em temperatura ambiente.

A revelação dos anticorpos foi realizada através da reação da enzima peroxidase visualizada pela coloração com DAB ((3-3)-tetrahidrocloreto de diaminabenzidina), contra-corados com hematoxilina da Haris e analisados em microscópio óptico (OLYMPUS BX40), nos aumentos 40X, 100X, 200X, 400X, por dois patologistas de forma "duplo cego".

\section{Análise imunoistoquímica}

A imunolocalização das proteínas p53 e p16 foi considerada positiva ou negativa de acordo com a quantidade de células imunomarcadas. Foi considerado p53 positivo quando a expressão nuclear ocorreu em quantidade igual ou superior a $10,00 \%$ 
das células imunocoradas. O p53 negativo foi obtido quando a expressão nuclear foi inferior a $10,00 \%$ das células imunomarcadas. Para p16 positivo consideramos expressão nuclear ou nuclear e citoplasmática, mas nunca apenas citoplasmática, em quantidade igual ou superior a 10,00\% das células imunomarcadas. Já o p16 foi considerado negativo quando a expressão nuclear ou nuclear e citoplasmática, mas nunca apenas citoplasmática, foi inferior a $10,00 \%$ das células imunomarcadas.

A imunoreatividade da COX-2 foi considerada positiva ou negativa, de acordo com a intensidade de coloração nas células. Considerou-se COX-2 negativo quando da ausência da expressão citoplasmática e COX-2 positivo quando da presença de expressão citoplasmática. Os casos COX-2 positivos foram categorizados de acordo com a intensidade de coloração em escores: escore $1+$, intensidade discreta da expressão citoplasmática; escore 2+, intensidade moderada da expressão citoplasmática; escore 3+, intensidade acentuada da expressão citoplasmática.

A leitura das lâminas para interpretação imunoistoquímica foi realizada de forma "duplo cego", por dois patologistas experientes da Consultoria em Patologia Dr. Carlos E. Bachi, Botucatu, SP.

\section{Análise estatística}

A análise estatística entre os resultados da imunorreatividade das proteínas p53, p16 e COX-2 e sua associação com o estádio do tumor foi obtida com o emprego do teste do qui ao quadrado de associação. A comparação da expressão das proteínas entre o tecido normal e o tecido tumoral empregou o teste exato de Fischer. Para todos os testes foi utilizado o software GraphPad Instat (versão 3.05, GraphPad Software Incorporation, San Diego, CA, EUA).

\section{RESULTADOS}

\section{Caracterização da amostra}

Foram estudados 31 casos de carcinoma de células escamosas, $25(80,64 \%)$ homens e $6(19,35 \%)$ mulheres, com média de idade de 55 anos. Em relação à localização, é possível dizer que a maioria dos tumores, 16 casos $(51,61 \%)$, foram encontrados no terço inferior do esôfago, seguido por 13 casos $(41,93 \%)$, no terço médio, 1 caso $(3,22 \%)$, no terço superior e 1 caso $(3,22 \%)$, nos terços médio e inferior, concomitantemente (Tabela 1).

A avaliação da diferenciação tumoral revelou 14 casos $(45,16 \%)$ moderadamente diferenciados, 12 (38,70\%) bem diferenciados e $5(16,12 \%)$ pouco diferenciados. Em 25 casos $(80,64 \%)$ não foi detectada invasão vascular e em $23(74,19 \%)$ não se observou invasão perineural (Tabela 1).

Segundo o estádio tumoral TNM, 13 casos (41,93\%) foram estádio IIA, $13(41,93 \%)$ foram estádio III, seguidos por $3(9,67 \%)$ estádio IIB e $2(6,45 \%)$ estádio I. Desta forma, os resultados indicam a predominância para os estágios IIA e III (Tabela1).

\section{Expressão imunoistoquímica de p53, p16 e COX-2}

A análise imunoistoquímica da hiperexpressão do p53 foi estatisticamente diferente do tecido normal $(P=0,0016)$ em 15 amostras tumorais $(48,38 \%$ ) (Tabela 2$)$.
TABELA 1. Características gerais das amostras de carcinoma de células escamosas de esôfago

\begin{tabular}{|c|c|c|}
\hline Característica & & $\mathrm{n}$ \\
\hline \multirow[t]{2}{*}{ Sexo } & Masculino & 25 \\
\hline & Feminino & 6 \\
\hline \multirow[t]{4}{*}{ Idade (anos) } & $<50$ & 11 \\
\hline & $51-60$ & 10 \\
\hline & $61-70$ & 7 \\
\hline & $>70$ & 3 \\
\hline \multicolumn{3}{|c|}{ Localização do tumor } \\
\hline & Terço superior & 1 \\
\hline & Terço médio & 13 \\
\hline & Terço inferior & 16 \\
\hline & Terço médio e inferior & 1 \\
\hline \multicolumn{3}{|c|}{ Grau de diferenciação } \\
\hline & Bem diferenciado & 12 \\
\hline & Moderadamente diferenciado & 14 \\
\hline & Pouco diferenciado & 5 \\
\hline \multicolumn{3}{|l|}{ Invasão vascular } \\
\hline & Presente & 6 \\
\hline & Ausente & 25 \\
\hline \multicolumn{3}{|c|}{ Invasão perineural } \\
\hline & Presente & 8 \\
\hline & Ausente & 23 \\
\hline \multicolumn{3}{|l|}{ Estádio } \\
\hline & I & 2 \\
\hline & IIA & 13 \\
\hline & IIB & 3 \\
\hline & III & 13 \\
\hline
\end{tabular}

TABELA 2. Reatividade imunoistoquímica da p53, p16 e COX-2 nas amostras de carcinoma de células escamosas de esôfago e tecido normal

\begin{tabular}{lccccccccc}
\hline & $(\mathrm{n})$ & \multicolumn{2}{c}{$\mathrm{p} 53$} & $\mathrm{p} 16$ & \multicolumn{3}{c}{ COX-2 } \\
\hline & & + & - & + & - & +1 & +2 & +3 \\
Tecido não-tumoral & 31 & 3 & 28 & 0 & 31 & 12 & 18 & 1 \\
Tecido tumoral & 31 & $15^{*}$ & 16 & 5 & 26 & 0 & 7 & $24^{* *}$ \\
\hline
\end{tabular}

* estatisticamente diferente do tecido normal $(P=0,0016)$

** estatisticamente diferente do tecido normal $(P<0,0001)$

Não foi detectada marcação imunoistoquímica estatisticamente diferente do tecido normal em relação ao p16 (Tabela 2).

$\mathrm{Na}$ análise estatística a coloração positiva para p53 e p16 foi considerada positiva, quando mais de $10,00 \%$ das células apresentavam coloração nuclear para p53, nuclear e citoplasmática para p16.

Foram evidenciados 24 amostras tumorais $(77,41 \%)$ positivos $3+$ para COX-2 estatisticamente diferentes do tecido normal não tumoral $(P<0,0001)$. Este comportamento não ocorreu em relação às intensidades de coloração $1+$ e $2+$ para COX-2 (Tabela 2).

$\mathrm{Na}$ análise estatística foi considerada como coloração positiva para COX-2 a presença de reatividade citoplasmática, de forma semiquantitativa e comparativa. A reatividade positiva para COX-2 foi classificada de acordo com a intensidade da presença da expressão citoplasmática de coloração em escores $1,2,3$, respectivamente, para intensidades leve, moderada e acentuada.

\section{Associação entre estádio e expressão dos marcadores p53, p16 e Cox 2}

De 31 carcinomas de células escamosas de esôfago, 15 foram p53 positivos e destes, a maioria, 7 casos $(46,66 \%)$ foi 
estádio III (Tabela 3). De 26 carcinomas de células escamosas negativos para $\mathrm{p} 16$, a maioria, 11 casos $(42,30 \%)$ foi estádio IIA (Tabela 3).

TABELA 3. Associação entre o estádio do carcinoma de células escamosas de esôfago e a expressão das proteínas p53, p16 e COX-2

\begin{tabular}{lccccc}
\hline & \multicolumn{3}{c}{ Estádio (n) } & \multirow{2}{*}{ Total } \\
\cline { 2 - 4 } & I & IIA & IIB & III & \\
\hline p53 & 1 & 5 & 2 & 7 & 15 \\
+ & 1 & 8 & 1 & 6 & 16 \\
- & & & & & \\
p16 & 0 & 2 & 0 & 3 & 5 \\
+ & 2 & 11 & 3 & 10 & 26 \\
- & & & & & \\
COX-2 & 2 & 4 & 1 & 0 & 7 \\
+2 & 0 & 9 & 2 & 13 & $24 *$ \\
+3 & & & & & \\
\hline
\end{tabular}

* existe associação significativa $(P=0,01)$

A análise estatística avaliando a associação entre os marcadores p53 e p16 com o estádio das amostras, não mostrou associação estatisticamente significante (Tabela 3 ).

Em relação à COX-2, das 24 amostras de tumor $\mathrm{COX}-23+$, $13(54,16 \%)$ foram estádio III (Tabela 3$)$.

A análise estatística correlacionando o marcador COX-2, escore $3+$, com estádio mostrou que existe associação estatisticamente significante $(P=0,01)$ (Tabela 3$)$.

\section{DISCUSSÃO}

O diagnóstico do câncer esofágico geralmente é realizado tardiamente, identificado pelo aparecimento da disfagia. Desta forma, a detecção é feita nas formas avançadas cuja sobrevida de 5 anos é inferior a 10\%. Alterações nos mecanismos moleculares que regulam a diferenciação, crescimento, invasão e morte celular determinam o comportamento maligno neoplásico, contribuindo para o estabelecimento e progressão dos tumores esofágicos. Neste sentido, a identificação de alterações moleculares complementará as informações histopatológicas e poderá trazer subsídios para a avaliação prognóstica dos tumores e pacientes. No presente estudo avaliou-se a existência de associação entre a expressão das proteínas p53, COX-2 e p16 com o estádio do carcinoma escamoso de esôfago ${ }^{(6)}$.

A caracterização geral da amostra revela que $80,64 \%$ dos casos estudados são relativos a pacientes do sexo masculino, com idade média de 56 anos. Esta distribuição está de acordo com o observado na literatura ${ }^{(6,10)}$.

De cinco amostras pobremente diferenciadas, quatro foram positivas para o marcador p53, colaborando para o fato de p53 ser um marcador de pior prognóstico, enquanto que de 26 amostras entre moderadamente e bem diferenciadas, 10 foram p53 positivas. Nos carcinomas de células escamosas bem diferenciadas, $100 \%$ foram p16 negativos, enquanto que $21,42 \%$ dos moderadamente diferenciados e $40 \%$ dos pouco diferenciados, foram $\mathrm{p} 16$ positivos.
Nos carcinomas de células escamosas pouco diferenciadas, $100 \%$ foram COX-2 positivo $3+, 64,28 \%$ dos moderadamente diferenciados e $83,33 \%$ dos bem diferenciados foram COX-2 positivo $3+$. A marcação de COX-2 positivo $2+$ ocorreu em $16,67 \%$ dos bem diferenciados, em $35,71 \%$ dos tumores moderadamente diferenciados e em nenhuma amostra pouco diferenciada.

Quanto ao estádio tumoral, 83,87\% dos casos estão no estágio $11 \mathrm{~A}$ e 111 . Destes, $46,15 \%$ foram positivos para o marcador $\mathrm{p} 53$. Para o marcador p16, 80,76\% dos casos foram negativos e $19,23 \%$ foram positivos. Para COX-2, $84,61 \%$ foi positivo $3+$ e $15,38 \%$ positivo $2+$.

Em mucosa normal de esôfago, pode-se determinar as diferenças de intensidade de coloração em relação à área tumoral, pois se observa que começa a aparecer a positividade 1+ para COX-2, embora nenhum dos casos tenha sido negativo para COX-2. Nas amostras não-tumorais, 38,71\% foram positivas com intensidade $1+$ para COX-2. Destas, $8,33 \%$ foram positivas para p53 e $91,66 \%$ negativos. De 58,06\% de casos com intensidade $2+, 94,44 \%$ foram negativos para p53 e 5,56\% positivos para p53. De 3,23\% com intensidade 3+ para COX-2, 100\% foram também positivos para p53. Não houve nenhum caso COX-2 positivo $3+$ e p53 negativo.

Em área tumoral, 48,38\% dos casos foram positivos para $\mathrm{p} 53$, enquanto que $90,32 \%$ das amostras de mucosa normal foram negativas para o $\mathrm{p} 53$, porém $9,67 \%$ das amostras não-tumorais foram p53 positivas. Tais observações são concordantes com estudos que apontam ser a oncoproteína p53 detectada por imunoistoquímica por ser produto de gene mutante. Mais ainda, ela só pode ser visualizada na forma mutante quando tem uma meia vida mais longa e tende a se acumular no núcleo, onde é visualizada. Entretanto, algumas situações especiais, como as que envolvem interações com proteínas virais, lesão celular ou proliferação celular, são capazes de alterar a estabilidade da proteína e, conseqüentemente, apresentar proteína p53 mesmo selvagem, detectável no estudo imunoistoquímico ${ }^{(1,5,15)}$.

O p53 é o principal gene alterado em tumor de esôfago, sendo que o carcinoma esofágico apresenta a segunda maior incidência de mutação neste gene, pois cerca de $40,00 \%$ dos carcinomas escamosos de esôfago possuem esta mutação ${ }^{(6)}$. Na presente investigação, 48,38\% das amostras tumorais apresentaram p53 mutante detectado pela presença da oncoproteína.

Em relação ao marcador p16, somente 16,12\% das amostras de tumor foram positivas para $\mathrm{p} 16$ e $84,37 \%$ foram $\mathrm{p} 16$ negativas, $\mathrm{o}$ que muito provavelmente ocorra pela alta agressividade do tumor. A hiperexpressão da 16 tem sido relacionada a melhor prognóstico para carcinoma de células escamosas, pois a hiperexpressão da p16 em área tumoral sugere que não houve hipermetilação do gene CDKN2A, que normalmente codifica esta proteína ${ }^{(29)}$. A maioria dos carcinomas escamosos de esôfago tem hipermetilação do gene CDKN2A e, por isto, 50\%-70\% deles não expressam p16, sendo isto um fator de pior prognóstico ${ }^{(9,19,25)}$.

O marcador COX-2 escore $3+$, com acentuada expressão citoplasmática, foi positivo em $100 \%$ dos casos em área tumoral, correspondendo este valor a 77,42\% COX-2 escore $3+$, enquanto que em mucosa adjacente apenas 3,23\% correspondem a COX-2 escore $3+$. Com isso, é possível sugerir que a intensidade mais acentuada de coloração 
para COX-2, ou seja, o escore $3+$ possa se relacionar com a presença do tumor. Segundo o estádio tumoral, apenas a expressão citoplasmática na intensidade acentuada da COX-2, ou seja, no escore $3+$ apresentou associação estatisticamente significante, o que está de acordo com os estudos que apontam a proteína COX-2 como indicadora de prognóstico no câncer esofágico $^{(12,14,16,23,24,30,32)}$.

\section{CONCLUSÕES}

Com base nos achados deste estudo, pode-se dizer que houve relação positiva entre a expressão de COX-2 escore 3+ e estádio mais avançado da doença, enquanto que as proteínas p53 e p16 não podem ser associadas ao estádio destes tumores, devendo ser mais amplamente estudadas em relação a outros parâmetros histopatológicos, tais como: invasão e tamanho do tumor.

Felin IPD, Grivicich I, Felin CR, Regner A, Rocha AB. p53, p16 E COX-2 expression in esophageal squamous cell carcinoma and histopathological association. Arq Gastroenterol. 2008;45(4):308-12.

ABSTRACT - Background - The esophageal carcinoma represents about 2\% of malignant tumors and is the third most common cause of gastrointestinal cancer. The correlation between immunohistochemistry markers, such as p53, p16 and COX-2 proteins and cancer esophageal prognosis has been suggested. Aims - To Investigate whether the expression of p53, p16 and COX-2 proteins are associated to tumor staging. Methods - For this purpose we proceeded immunohistochemistry assays and TMN in 31 esophageal tumor and normal tissue samples. The p53 nuclear expression was considered positive when it appears in $10.00 \%$ or more cells. COX-2 expression was scored according to intensity in three scores $(1+, 2+, 3+)$. Results - On the tumor samples the results presented $48.38 \%$ positivity for $\mathrm{p} 53,16.12 \%$ for $\mathrm{p} 16$ and $100 \%$ with $1+, 2+$ or $3+$ scores for COX- 2 . However, when we investigated whether the expression of p53, p16 and COX-2 proteins are related to tumor staging, only COX-2 expression, score 3+, had shown statistical significant association. Conclusion - Therefore, in the present study we could see positive correlation between COX-2 protein and high grade tumor as well as advanced tumor staging in esophageal carcinoma.

HEADINGS - Esophageal neoplasms. Carcinoma, squamous cell. Tumor suppressor protein p53. Cyclin-dependent kinase inhibitor p16. Cyclooxygenase 2.

\section{REFERÊNCIAS}

1. Alberts B, Bray D, Lewis J, Raff M, Roberts K, Watson JD. Biologia molecular da célula. $4^{\mathrm{a}}$ ed. Porto Alegre: Artmed; 2004. p.1255-90.

2. Alves VFA, Bacchi CE, Vassallo J. Manual de imuno-histoquímica. São Paulo: Sociedade Brasileira de Patologia; 1999. p.237-57.

3. Bacchi CE, Almeida PCC, Franco M. Manual de padronização de laudos histológicos.

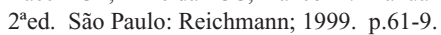

4. Brentani MM, Coelho FRG, Kowalski LP. Bases da oncologia. $2^{\text {a }}$ ed. São Paulo: Lemor; 2003. p.136-45.

5. Chetty R, Simelane S. p53 and cyclin a protein expression in squamous carcinoma of the oesophagus. Pathol Oncol Res. 1999;5:193-6.

6. Cotran RS, Kumar V, Collins T, editores. Robbins patologia estrutural e funcional. $7^{\mathrm{a}}$ ed. Rio de Janeiro: Guanabara Koogan; 2006. p.281-356; 846-50.

7. Enzinger PC, Mayer RJ. Esophageal cancer. N Engl J Med. 2003;349: 2241-52.

8. Ferreira CG, Rocha JC. Oncologia molecular. São Paulo: Atheneu; 2004. p.15564.

9. Fujiwara S, Noguchi T, Takeno S, Kimura Y, Fumoto S, Kawahara K. Hypermethylation of p16 gene promoter correlates with loss of p16 expression that results in poorer prognosis in esophageal squamous cell carcinomas. Dis Esophagus. 2008;21:125-31.

10. Guimarães JR. Manual de oncologia. $2^{\text {a }}$ ed. São Paulo: Libbs Farmacêutica; 2006. p.417-23.

11. Hsu SM, Raine L, Fanger H. Use of avidin-biotin peroxidase complex (ABC) in immunoperoxidase techniques: a comparison between $\mathrm{ABC}$ and unlabelled antibody (PAP) procedures. J Histochem Cytochem. 1981;29:577-80.

12. Ikeda G, Isaji S, Chandra B, Watanabe M, Kawarada Y. Prognostic significance of biologic factors in squamous cell carcinoma of the esophagus. Cancer. 1999;86:1396405 .

13. Iodish H, Berk A, Zipursky SL, Matsudaira P, Baltimore D, Darnell JE. Biologia celular e molecular. $4^{\mathrm{a}}$ ed. Rio de Janeiro: Revinter; 2002. p.1054-84.

14. Kawabe A, Shimada Y, Uchida S, Maeda M, Sato F, Itami A, Imamura M. Expression of cyclooxigenase-2 is associated with carcinogenesis of the lower part of thoracic esophageal squamous cell carcinoma and p53 expression. Oncology. 2002;62:4654.

15. Klumb CE, Cavalcanti Júnior GB. Avaliação dos métodos de detecção das alterações do gene e proteína p53 nas neoplasias linfóides. Rev Bras Hematol Hemoter. 2002;24:111-25.

16. Kuo KT, Chow KC, Wu IC, Lin CS, Wang HW, Li WY, Wang LS. Clinicopathologic significance of cyclooxigenase-2 overexpression in esophageal squamous cell carcinoma. Ann Thorac Surg. 2003;76:909-14.

17. Lee RG, Compton CC. Protocol for the examination of specimens removed from patients with esophageal carcinoma. A basis for checklists. The Cancer Committee,
College of American Pathologists, and the Task Force on the Examination of Specimens From Patients With Esophageal Cancer. Arch Pathol Lab Med. 1997;121:925-9.

18. Lehrbach DM, Nita ME, Cecconello I. Aspectos moleculares da carcinogênese do carcinoma epidermóide do esôfago. Arq Gastroenterol. 2003;40:256-61.

19. Louro ID, Llerena JC, Mello MS, Prolla P, Froes N. Genética molecular do câncer. 2nd ed. São Paulo: MSG; 2002. p.63-90.

20. Mir L. Genômica. São Paulo: Atheneu; 2004. p.363-85.

21. Philip AP, Ajani J. Carcinoma of the esophagus. Medical oncology: a comprehensive review. [Disponível em: http://www.cancernetwork.com/textbook/morev14.htm] Acesso: set 202004.

22. Pinho MSL. Biologia molecular do câncer: fundamentos para a prática médica. Rio de Janeiro: Revinter; 2005. p.83-4.

23. Ratnasinghe D, Tangrea J, Roth MJ, Dawsey SM, Hu N, Anver M, Wang O, Taylor PR. Expression of cyclooxygenase-2 in human squamous cell carcinoma of the esophagus; an immunohistochemical survey. Anticancer Res. 1999;19:171-4.

24. Shamma A, Yamamoto H, DokiY, Okami J, Kondo M, Fujiwara Y, Yano M, Inoue M, Matsuura N, Shiozaki H, Monden M. Up-regulation of cyclooxigenase-2 in squamous carcinogenesis of the esophagus. Clin Cancer Res. 2000;6:1229-38.

25. Shimada Y, Imamura M, Watanabe G, Uchida S, Harada H, Makino T, Kano M. Prognostic factors of oesophageal squamous cell carcinoma from the perspective of molecular biology. Br J Cancer 1999; 80:1281-8.

26. Simon SD. A biologia molecular aplicada ao diagnóstico e tratamento do câncer. Diálogo Científico. 2004;1:18-22.

27. Sobin $\mathrm{LH}$, wittekind $\mathrm{CH}$. Classification of malignant tumours. International Union Against Cancer. 6th ed. Whiley-Liss; 2004. p.64-8.

28. Sternberg S, Antonioli D, Carter D, Mills S, Oberman H. Diagnostic surgical pathology. 3rd ed. Philadelphia: Lippincott Williams \& Wilkins; 1999. p.1283-309.

29. Sturm I, Petrowsky H, Volz R, Lorenz M, Radetzki S, Hillebrand T, Wolff G, Hauptmann S, Dorken B, Daniel PT. Analysis of p53/BAX/p16 (inK4a/CDKN2) in esophageal squamous cell carcinoma: high BAX and p16 identifies patients with good prognosis. J Clin Oncol. 2001;19:2272-81.

30. Yu HP, Xu SQ, Liu L, Shi LY, Cai XK, Lu WH, Lu B, Su YH, LiYY. Cyclooxigenase-2 expression in squamous dysplasia and squamous cell carcinoma of the esophagus. Cancer Lett. 2003;198:193-201.

31. Zhang H, Chen SH, Li YM. Epidemiological investigation of esophageal carcinoma World J Gastroenterol. 2004;10:1834-5.

32. Zimmermann KC, Sarbia M, Weber AA, Borchard F, Gabbert HE, SchrörK Cyclooxygenase-2 expression in human esophageal carcinoma. Cancer Res. 1999;59:198-204

Recebido em 30/1/2008 Aprovado em 26/3/2008 